\title{
A New RTL Design Approach for a DCT/IDCT-Based Image Compression Architecture using the mCBE Algorithm
}

\author{
Rachmad Vidya Wicaksana Putra, Rella Mareta, Nurfitri Anbarsanti \\ \& Trio Adiono
}

IC Design Laboratory, Electrical Engineering, School of Electrical Engineering and Informatics, Institut Teknologi Bandung, Jalan Ganesha 10, Bandung 40132, Indonesia

Email: rachmadvidyawp@ students.itb.ac.id, rachavidyawp@gmail.com

\begin{abstract}
In the literature, several approaches of designing a DCT/IDCT-based image compression system have been proposed. In this paper, we present a new RTL design approach with as main focus developing a DCT/IDCT-based image compression architecture using a self-created algorithm. This algorithm can efficiently minimize the amount of shifter-adders to substitute multipliers. We call this new algorithm the multiplication from Common Binary Expression (mCBE) Algorithm. Besides this algorithm, we propose alternative quantization numbers, which can be implemented simply as shifters in digital hardware. Mostly, these numbers can retain a good compressed-image quality compared to JPEG recommendations. These ideas lead to our design being small in circuit area, multiplierless, and low in complexity. The proposed 8-point 1D-DCT design has only six stages, while the 8-point 1D-IDCT design has only seven stages (one stage being defined as equal to the delay of one shifter or 2-input adder). By using the pipelining method, we can achieve a high-speed architecture with latency as a trade-off consideration. The design has been synthesized and can reach a speed of up to $1.41 \mathrm{~ns}$ critical path delay $(709.22 \mathrm{MHz})$.
\end{abstract}

Keywords: DCT/IDCT architecture; low complexity; mCBE Algorithm; multiplierless; new RTL design approach.

\section{$1 \quad$ Introduction}

Many kinds of digital image and video processing techniques have been proposed in the literature. Most of them, require discrete cosine transform (DCT). In this paper, we will discuss DCT-based image compression, one of the most interesting topics in image processing. Actually, DCT is not an algorithm specifically developed for image compression. But, we can take advantage of DCT theory to support well-performing image compression [1].

In the past years, many DCT-based researches have been conducted. Loeffler, C., et al. [2] proposed a low complexity DCT using the Flow-Graph Algorithm. This design requires 11 multiplications and 29 additions. Jeong, et al. [3]

Received November $14^{\text {th }}, 2011$, Revised February $26^{\text {th }}, 2012$, Accepted for publication September $22^{\text {nd }}, 2012$. Copyright (C) 2012 Published by LPPM ITB,ISSN: 1978-3086, DOI: 10.5614/itbj.ict.2012.6.2.3 
proposed a low-power multiplierless DCT architecture using image data correlation (cordic). Ruiz, et al. [4] proposed a high-throughput parallel-pipeline 2D-DCT/IDCT processor. Heyne, et al. [5] proposed a combination of the Loeffler DCT and cordic method, which requires 38 adders and 16 shifters for 8-point 1D-DCT and consists of 3 cordic blocks with several adders. Each block consists of an iterative shift-add operation and the longest path takes three iterations. Therefore, its longest path has an 8-stage delay (we define one stage as the delay of one shifter or 2-input adder). Byoung-Il Kim, et al. [6] have proposed a low-power multiplierless DCT for image/video coders. Subramanian, et al. [7] have proposed a VLSI implementation of a fully pipelined multiplierless 2D-DCT/IDCT architecture for JPEGs.

In this paper, we propose a new approach of designing a DCT/IDCT-based image compression architecture. The main focus of our research was to develop the architecture by using a self-created algorithm that can efficiently minimize the amount of shifter-adders to substitute constant multipliers. We named it: multiplication from Common Binary Expression (mCBE) Algorithm. We also propose alternative quantization numbers, which can be implemented simply as shifters. Mostly, these numbers can retain a good compressed image quality compared to JPEG recommendations. We had three objectives. Firstly, the design had to be able to operate well, producing a good compressed image quality represented by Peak-to-peak Signal to Noise Ratio (PSNR) and Mean Square Error (MSE) values. Secondly, the design had to have less than 8-stage delay of flow-graph architecture without iterations for 8-point 1D-DCT/IDCT. Thirdly, the design had to have a small circuit area, be multiplierless, and have low complexity, so that it can be implemented easily in digital hardware.

This paper is organized in a number of sections. First, the introduction section briefly introduces: (1) the importance of DCT theory in image compression systems, (2) several past researches about DCT-based image compression systems, and (3) the main points of the proposed design. This section is followed by a brief explanation of DCT/IDCT theory and its usage in image compression systems. The next section is about the proposed design. Here, we explain our design approach and its implementation in detail. This section is followed by the synthesis and analysis regarding our proposal. We also compare the proposed design with other design methods. In the following section, we explain the simulation and FPGA implementation. Finally, this section is followed by conclusion and references, respectively.

\section{Fundamental Theory}

In designing a DCT/IDCT-based image compression system, we need to use DCT/IDCT formulations and quantification-dequantification processing [8]. 


\subsection{Discrete Cosine Transform Formulation}

The equation of 1-Dimension N-points DCT is formulated as:

$$
Z(u)=\alpha(u) \sum_{x=0}^{N-1}\left(f_{(x)} \cos \left[\frac{\pi(2 x+1) u}{2 N}\right]\right), \alpha(u)=\left\{\begin{array}{l}
\sqrt{\frac{1}{N}} \text { for } u=0 \\
\sqrt{\frac{2}{N}} \text { for } u \neq 0
\end{array}\right.
$$

\subsection{Inverse Discrete Cosine Transform Formulation}

The equation of 1-Dimension N-points Inverse DCT is formulated as:

$$
f(x)=\sum_{x=0}^{N-1} \alpha(u)\left(Z_{(u)} \cos \left[\frac{\pi(2 x+1) u}{2 N}\right]\right), \alpha(u)=\left\{\begin{array}{l}
\sqrt{\frac{1}{N}} \text { for } u=0 \\
\sqrt{\frac{2}{N}} \text { for } u \neq 0
\end{array}\right.
$$

\subsection{Basic DCT/IDCT-Based Image Compression System}

The main idea behind this image compression system is to reduce the image data size without significantly reducing image quality to the human eye. This technique will remove the least significant (high-frequency) information data from the image. The most significant information data usually have a low frequency. For the human eye it is more difficult to see differences in high frequencies than in low frequencies. Therefore, by eliminating the higher frequencies we can significantly reduce the image size without abandoning image quality.

The input and output data of the DCT/IDCT-based compression system can be provided by using the $\mathrm{YCbCr}$ format. Input image data in JPEG format are converted to $\mathrm{YCbCr}$ format, which has three macroblock components: $\mathrm{Y}$ (luminance), $\mathrm{Cb}$ (chrominance blue), and $\mathrm{Cr}$ (chrominance red). Each component is computed based on microblock segmentation (8x8pixels), as shown in Figure 1. The inverse process is applied to the output data of the system in order to get the actual image (JPEG format).Therefore, by combining Eqs. (1) and (2) with microblock characters, we can compute 64-point data in a 2D-DCT/IDCT process from an 8-point input 1D-DCT only.

Figure 2 shows the details of the process of the complete compression system. In the complete compression system, first the image data will be represented as spatial domain data. These data will be transformed to frequency domain data using 2D-DCT processing. Then, we organize the frequency domain data in term of importance. Thus, we can eliminate the high-frequency data using 
quantification. Next, we do 2D-IDCT processing, which is the inversion of 2DDCT processing, to get the compressed image data in the spatial domain.

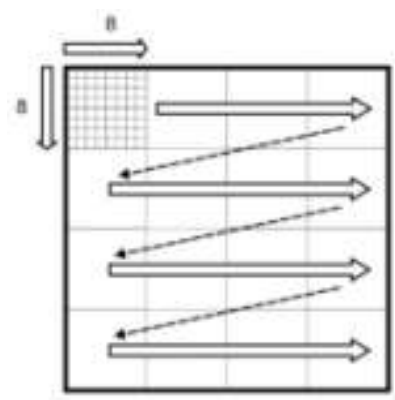

Figure 1 Microblock segmentation (8x8-pixels).

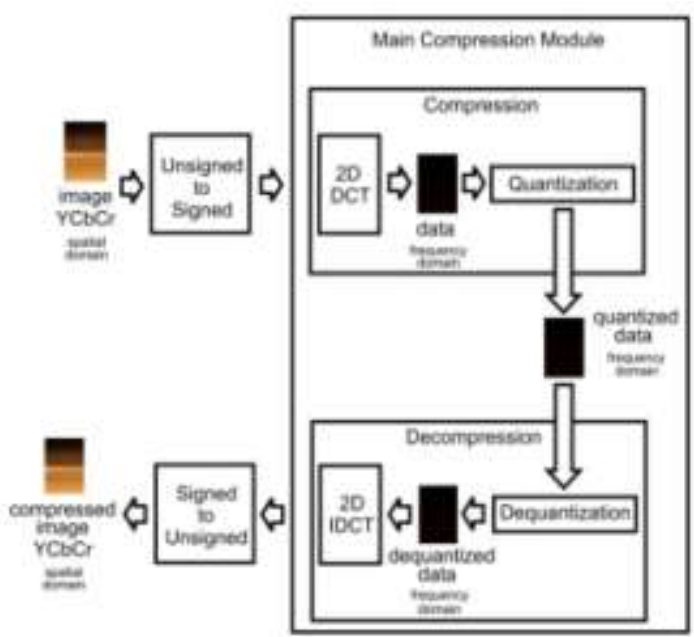

Figure 2 Detail of complete compression system.

\subsection{Quantization and Dequantization}

The other important modules in this compression system are quantization and dequantization. The quantization module performs the quantification process on the microblock data. The dequantization module performs the dequantification process on the microblock data. The general equation for quantization is:

$$
Q_{D C T}=\text { round }\left(\frac{D C T(u, v)}{Q(u, v)}\right)
$$

Dequantization is the inverse of quantization. From equation (3), we have $\operatorname{DCT}(u, v)$ and $Q(u, v)$ as input for the quantification process. Parameters $u$ and $v$ represent row and column location. DCT $(\mathrm{u}, \mathrm{v})$ represents the image data location in a microblock (8x8pixels) and $\mathrm{Q}(\mathrm{u}, \mathrm{v})$ represents the quantizer numbers in the quantization matrices (denominators of the quantification process). There is no fixed numbers of quantization matrices. It is the prerogative of the user to select the quantization matrices. However, the JPEG committee has recommended a number of quantization matrices ( $\mathrm{Q}$ matrices), for example, $\mathrm{Q}_{50 \_ \text {LUMINANCE }}$ and $\mathrm{Q}_{50 \_ \text {CHROMINANCE: }}$ 


$Q_{50 \_ \text {LUMINANCE }}=\left[\begin{array}{cccccccc}16 & 11 & 10 & 16 & 24 & 40 & 51 & 61 \\ 12 & 12 & 14 & 19 & 26 & 58 & 60 & 55 \\ 14 & 13 & 16 & 24 & 40 & 57 & 69 & 56 \\ 14 & 17 & 22 & 29 & 51 & 87 & 80 & 62 \\ 18 & 22 & 37 & 56 & 68 & 109 & 103 & 77 \\ 24 & 35 & 55 & 64 & 81 & 104 & 113 & 92 \\ 49 & 64 & 78 & 87 & 103 & 121 & 120 & 101 \\ 72 & 92 & 95 & 98 & 112 & 100 & 103 & 99\end{array}\right] \quad Q_{50 \_ \text {CHROMINANCE }}=\left[\begin{array}{cccccccc}17 & 18 & 24 & 47 & 99 & 99 & 99 & 99 \\ 18 & 21 & 26 & 66 & 99 & 99 & 99 & 99 \\ 24 & 26 & 56 & 99 & 99 & 99 & 99 & 99 \\ 47 & 66 & 99 & 99 & 99 & 99 & 99 & 99 \\ 99 & 99 & 99 & 99 & 99 & 99 & 99 & 99 \\ 99 & 99 & 99 & 99 & 99 & 99 & 99 & 99 \\ 99 & 99 & 99 & 99 & 99 & 99 & 99 & 99 \\ 99 & 99 & 99 & 99 & 99 & 99 & 99 & 99\end{array}\right]$

\section{Proposed Design}

\subsection{Choosing the Image Compression System}

In this proposal, we have used the basic system that is shown in Figure 2. Here, we have defined two subparts of the complete compression system: the supporting modules and the main compression module. The supporting modules consist of unsigned-to-signed and signed-to-unsigned modules. The main compression module consists of three main parts: a 8-point 1D-DCT block with transposition buffer, a 8-point 1D-IDCT block with transposition buffer, and Quantization-Dequantization blocks, as shown in Figure 3-4.

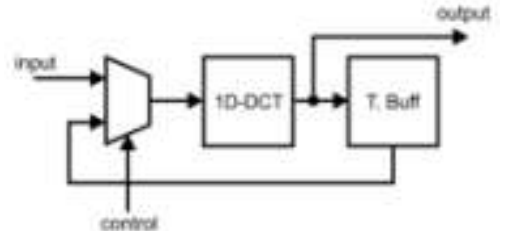

Figure 3 Basic architecture in 2D-DCT. Figure 4 Basic architecture in 2D-IDCT.

This design approach was chosen in order to achieve the stated objectives, specifically the small area and low complexity. We did not choose a direct 2DDCT/IDT architecture, because of its huge area. Each 2D-DCT/IDCT design uses a transposition buffer to complete the $2 \mathrm{D}$ process. The area of the proposed design is smaller than the area of a direct 2D-DCT/IDCT. Obviously, our design needs more cycles to compare the process.

\subsection{Basic DCT/IDCT Matrices Equations}

We explore DCT/IDCT equations in Eqs. (1) and (2) to arrange the DCT/ IDCT matrices equations. The obtained matrices equations from the 8-point DCT process are shown in Eqs. (4) and (5), which are similar to the matrices equations from reference [9]. From these equations, we find a simple design for the digital hardware architecture. 


$$
\begin{aligned}
& {\left[\begin{array}{c}
z_{0} \\
z_{2} \\
z_{4} \\
z_{6}
\end{array}\right]=\frac{1}{\sqrt{8}}\left[\begin{array}{cccc}
1 & 1 & 1 & 1 \\
b & e & -e & -b \\
1 & -1 & -1 & 1 \\
e & -b & b & -e
\end{array}\right]\left[\begin{array}{l}
x_{0}+x_{7} \\
x_{1}+x_{6} \\
x_{2}+x_{5} \\
x_{3}+x_{4}
\end{array}\right]} \\
& {\left[\begin{array}{c}
z_{1} \\
z_{3} \\
z_{5} \\
z_{7}
\end{array}\right]=\frac{1}{\sqrt{8}}\left[\begin{array}{cccc}
a & -c & d & -f \\
c & f & -a & d \\
d & a & f & -c \\
f & d & c & a
\end{array}\right]\left[\begin{array}{c}
x_{0}-x_{7} \\
x_{6}-x_{1} \\
x_{2}-x_{5} \\
x_{4}-x_{3}
\end{array}\right]} \\
& a=\sqrt{2} \cos \left(\frac{\pi}{16}\right) \quad b=\sqrt{2} \cos \left(\frac{\pi}{8}\right) \\
& d=\sqrt{2} \cos \left(\frac{5 \pi}{16}\right) \quad e=\sqrt{2} \cos \left(\frac{3 \pi}{8}\right) \quad f=\sqrt{2} \cos \left(\frac{7 \pi}{16}\right)
\end{aligned}
$$

We use several symbols in the DCT matrices equations (4) and (5). A brief explanation of the symbols:

- Input of 8-point DCT process

- Output of 8-point DCT process

- Variables

$$
\begin{aligned}
& : x_{0}, x_{1}, x_{2}, x_{3}, x_{4}, x_{5}, x_{6}, x_{7} \\
& : z_{0}, z_{1}, z_{2}, z_{3}, z_{4}, z_{5}, z_{6}, z_{7} \\
& : a, b, c, d, e, f
\end{aligned}
$$

By using a similar idea, we also get the IDCT matrices equations. A brief explanation of symbols used in IDCT process:

- Input of 8-point IDCT process

- Output of 8-point IDCT process

- Variables

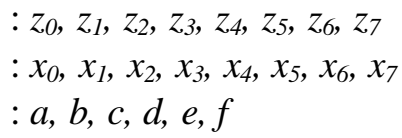

We will use the variables and expressions from this section for further exploration.

\subsection{Extracting the DCT/IDCT Matrices Equations}

Based on DCT matrices Eqs. (4) and (5), we can build several independent matrices equations. These are the extracted matrices equations:

$$
\left[\begin{array}{l}
z_{0} \\
z_{4}
\end{array}\right]=\left[\begin{array}{cc}
\frac{1}{\sqrt{8}} & \frac{1}{\sqrt{8}} \\
-\frac{1}{\sqrt{8}} & \frac{1}{\sqrt{8}}
\end{array}\right]\left[\begin{array}{l}
\left(x_{1}+x_{6}\right)+\left(x_{2}+x_{5}\right) \\
\left(x_{0}+x_{7}\right)+\left(x_{3}+x_{4}\right)
\end{array}\right]
$$




$$
\begin{aligned}
& {\left[\begin{array}{l}
z_{2} \\
z_{6}
\end{array}\right]=\frac{1}{\sqrt{8}}\left[\begin{array}{cc}
b & -e \\
e & b
\end{array}\right]\left[\begin{array}{l}
\left(x_{0}+x_{7}\right)-\left(x_{4}+x_{3}\right) \\
\left(x_{2}+x_{5}\right)-\left(x_{1}+x_{6}\right)
\end{array}\right]} \\
& {\left[\begin{array}{l}
z_{2} \\
z_{6}
\end{array}\right]=\left[\begin{array}{cc}
B & -E \\
E & B
\end{array}\right]\left[\begin{array}{l}
\left(x_{0}+x_{7}\right)-\left(x_{4}+x_{3}\right) \\
\left(x_{2}+x_{5}\right)-\left(x_{1}+x_{6}\right)
\end{array}\right]} \\
& {\left[\begin{array}{l}
p_{0} \\
p_{1}
\end{array}\right]=\frac{1}{\sqrt{8}}\left[\begin{array}{cc}
a & -f \\
f & a
\end{array}\right]\left[\begin{array}{l}
x_{0}-x_{7} \\
x_{4}-x_{3}
\end{array}\right]=\left[\begin{array}{cc}
A & -F \\
F & A
\end{array}\right]\left[\begin{array}{l}
x_{0}-x_{7} \\
x_{4}-x_{3}
\end{array}\right]} \\
& {\left[\begin{array}{l}
p_{2} \\
p_{3}
\end{array}\right]=\frac{1}{\sqrt{8}}\left[\begin{array}{cc}
f & a \\
-a & f
\end{array}\right]\left[\begin{array}{l}
x_{2}-x_{5} \\
x_{6}-x_{1}
\end{array}\right]=\left[\begin{array}{cc}
F & A \\
-A & F
\end{array}\right]\left[\begin{array}{l}
x_{2}-x_{5} \\
x_{6}-x_{1}
\end{array}\right]} \\
& {\left[\begin{array}{l}
q_{0} \\
q_{1}
\end{array}\right]=\frac{1}{\sqrt{8}}\left[\begin{array}{cc}
d & -c \\
c & d
\end{array}\right]\left[\begin{array}{l}
x_{2}-x_{5} \\
x_{6}-x_{1}
\end{array}\right]=\left[\begin{array}{cc}
D & -C \\
C & D
\end{array}\right]\left[\begin{array}{l}
x_{2}-x_{5} \\
x_{6}-x_{1}
\end{array}\right]} \\
& {\left[\begin{array}{l}
q_{2} \\
q_{3}
\end{array}\right]=\frac{1}{\sqrt{8}}\left[\begin{array}{ll}
d & -c \\
c & d
\end{array}\right]\left[\begin{array}{l}
x_{0}-x_{7} \\
x_{4}-x_{3}
\end{array}\right]=\left[\begin{array}{ll}
D & -C \\
C & D
\end{array}\right]\left[\begin{array}{l}
x_{0}-x_{7} \\
x_{4}-x_{3}
\end{array}\right]} \\
& z_{1}=p_{0}+q_{0} \\
& z_{3}=p_{3}+q_{3} \\
& z_{5}=p_{2}+q_{2} \\
& z_{7}=p_{1}+q_{1}
\end{aligned}
$$

From these equations, there are several inputs multiplied by several constant multipliers, which can be classified as three blocks of multiplications, as shown in Table 1.

Table 1 Three blocks of multiplications.

\begin{tabular}{ccc}
\hline $\begin{array}{c}\text { Blocks } \\
\text { Name }\end{array}$ & $\begin{array}{c}\text { Multiplicands } \\
\text { (Input Signals) }\end{array}$ & Multipliers \\
\hline $\mathrm{mCBE}_{0}$ & $\left(x_{1}+x_{6}\right)+\left(x_{2}+x_{5}\right),\left(x_{0}+x_{7}\right)+\left(x_{3}+x_{4}\right)$ & $\frac{1}{\sqrt{8}}$ \\
$\mathrm{mCBE}_{1}$ & $\left(x_{0}+x_{7}\right)-\left(x_{4}+x_{3}\right),\left(x_{2}+x_{5}\right)-\left(x_{1}+x_{6}\right)$ & $B, E$ \\
$\mathrm{mCBE}_{2}$ & $x_{0}-x_{7}, x_{4}-x_{3}, x_{2}-x_{5}, x_{6}-x_{1}$ & $A, C, D, F$ \\
\hline
\end{tabular}

\subsection{Fixed-Point Data Format}

We used a 20-bit data word-length, which consists of a 1-bit sign bit, 12bits of decimal data, and 7bits of fractional data. We chose a 12-bit data for decimal data because this accommodates the highest value that the compression process can reach. We chose a 7-bit data for fractional data because this is the shortest bit-length with a good performance in retaining image quality (high PSNR and low MSE value from modeling results, as shown in Figure 5). For the constant multipliers, we also used a 7-bit data as shown in Table 2. 


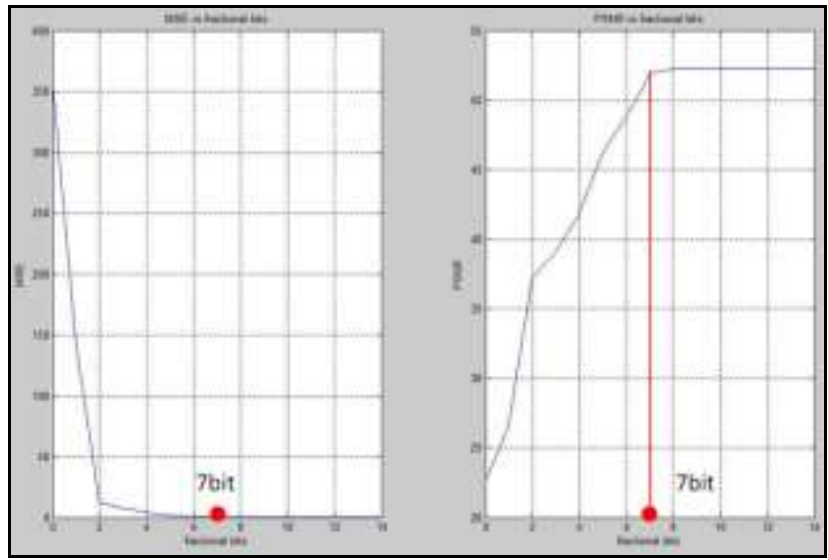

Figure 5 Modeling result of fractional bit length for MSE and PSNR.

Table 2 Constant multipliers bit representation.

\begin{tabular}{cccc}
\hline \multirow{2}{*}{ Variables } & \multirow{2}{*}{$\begin{array}{c}\text { Floating-Point } \\
\text { Value }\end{array}$} & \multicolumn{2}{c}{$\begin{array}{c}\text { Bit Representation } \\
\text { Approximation }\end{array}$} \\
\cline { 3 - 4 } & & Decimal & Fractional \\
\hline 1 & 0.3535534 & - & 0101101 \\
$\sqrt{8}$ & 0.49039264 & - & 0111111 \\
$A$ & 0.46193766 & - & 0111011 \\
$B$ & 0.415734806 & - & 0110101 \\
$C$ & 0.277785116 & - & 0100100 \\
$D$ & 0.191341716 & - & 0011000 \\
$E$ & 0.097545161 & - & 0001100 \\
$F$ & & & \\
\hline
\end{tabular}

\subsection{Multiplication Decomposition Using the mCBE Algorithm}

It follows from Section 3.3 that we need multiplication by several constant multipliers. We know that multiplication can be substituted by a shift-add operation. Thus, we propose an algorithm to minimize the amount of shifteradder used to substitute the multiplication, which uses several constant multipliers. This is the multiplication from Common Binary Expression (mCBE) Algorithm.

\begin{tabular}{|c|c|c|c|c|}
\hline \multirow{2}{*}{ L.isting } & \multicolumn{2}{|c|}{ MSB } & \multicolumn{3}{r|}{ LSB } \\
\hline & bit-n & $\ldots$ & bit-1 & bit-1) \\
\hline$C_{1}$ & & $V_{y}$ & & \\
\hline$C_{2}$ & & \\
\hline$C_{3}$ & & & & \\
\hline$\ldots$ & & & & \\
\hline$C_{B}$ & & & $H V$ & \\
\hline
\end{tabular}

Figure 6 Illustration of the mCBE algorithm design. 
Here, we present the mCBE Algorithm:

i. Listing of all constants $\left(\mathrm{C}_{1-\mathrm{n}}\right)$ which are used to multiply the same input, then produce a table as shown in Figure 6.

ii. Choose and classify every constant which has bit value 1 , more or equal to $70 \%$ from its bit's amount. There will be two groups:

a. Group of $[\geq 70 \%]$.

b. Group of $[<70 \%]$.

iii. Suppose there are 2 groups.

a. Group of $[\geq 70 \%]$ : Group A.

a.1 If only one constant fits in Group A, then:

$\checkmark$ Find out the most significant bit that contains the value of 1 .

$\checkmark$ If the position is bit-m, choose bit- $(\mathrm{m}+1)$ as MostShiftBit.

$\checkmark$ Then, determine the SubShiftBit to complete the substraction operation in order to get the desired result.

a.2 If there are several constants that fit in Group A, then:

$\checkmark$ For every constant, find out the most significant bit that contains the value of 1 .

$\checkmark$ If the position is bit-m, choose bit-(m+1) as MostShiftBit.

$\checkmark$ Then, determine the SubShiftBit to complete the substraction operation in order to get the desired result.

$\checkmark$ If it is possible to use the same MostShiftBit or SubShiftBit, then use them together to fit other constants.

b. Group of $[<70 \%]$ : Group B.

b.1 Find out the most appeared value of 1 for $V v$ (vertical view), and call it VvShiftBit. If there are several bits that fit, choose one.

b.2 From the chosen VvShiftBit point, find the most appeared bit value of 1 for the corresponding $H v$ (horizontal view). We call this bit HvShiftBit. There are three possible conditions: single HvShiftBit found, several HvShiftBits found, or no HvShiftBit found.

b.2.1 If there is a single HvShiftBit, then:

$\checkmark$ Choose this bit as AddShiftBit to the chosen VvShiftBit.

$\checkmark$ Then, still with the same VvShiftBit, check again from step (b.2) for unselected bits.

b.2.2 If there are several HvShiftBits, then:

Check whether between those HvShiftBits (Two or more $H v$ ShiftBits are grouped as $H v$ ShiftBitsG) there is a mutual inter- $H v$ relation (in the same constant).

$\checkmark$ If yes, then:

Choose the bit which has the least inter- $H v$ relation in $H v$ ShiftBitsG as AddShiftBit to the chosen VvShiftBit.

$\checkmark$ If $n o$, then:

Choose one as AddShiftBit to the chosen VvShiftBit. 


\section{b.2.3 If there is no HvShiftBit, then:}

$\checkmark$ Continue to step (iv).

iv. Loop from step (iii) until all values 1 are represented as MostShiftBit, VvShiftBit, SubShiftBit, or AddShifBit.

v. Design a butterfly-like flow-graph using chosen MostShiftBit, VvShiftBit, SubShiftBit, or AddShifBit.

Notes:

$\mathrm{Ci} \quad$ : Constant multiplier number $i$.

Vv : Vertical view relation.

$H v \quad$ : Horizontal view relation.

MostShiftBit : Biggest shifting bit value: its shifting result will be substracted from another signal (usually SubShiftBit).

$V v$ ShiftBit : Most appeared bit value 1 in vertical bit order, which is used as shifting bit.

$H v$ ShiftBit $\quad$ : Most appeared bit value 1 in inter- $H v$ relation to VvShiftBit.

HvShiftBitsG : Group of several HvShiftBits.

AddShiftBit : The bit which is chosen as shifter bit and its shifting output will be added to the result of VvShiftBit or another AddShiftBit output.

SubShiftBit : The bit which can be partner of MostShiftBit to complete the substraction operation.

\subsection{Implementation of the mCBE Algorithm}

\subsubsection{Basic mCBE Building Blocks}

Implementation of the mCBE Algorithm occurs inside the multiplication block. From Table 1, we get three blocks $\left(\mathrm{mCBE}_{0}, \mathrm{mCBE}_{1}\right.$, and $\left.\mathrm{mCBE}_{2}\right)$.

\section{a. The mCBE $_{0}$ Block}

Table 3 shows an illustration of the $\mathrm{mCBE}_{0}$ block design.

Table 3 Constant bit representation.

\begin{tabular}{|l|c|c|c|c|c|c|c|}
\hline \multirow{2}{*}{ Variables } & \multicolumn{7}{|c|}{ Bit Representation } \\
\cline { 2 - 7 } & $\mathbf{6}$ & $\mathbf{5}$ & $\mathbf{4}$ & $\mathbf{3}$ & $\mathbf{2}$ & $\mathbf{1}$ & $\mathbf{0}$ \\
\hline$\frac{1}{\sqrt{8}}$ & 0 & 1 & 0 & 1 & 1 & 0 & 1 \\
\hline & & 1 & & 1 & 1 & 0 & 1 \\
\hline
\end{tabular}

VvShiftBit ${ }_{0-1}$ AddShiftBit ${ }_{0-1}$

Then, we get the following architecture (Figure 7): 


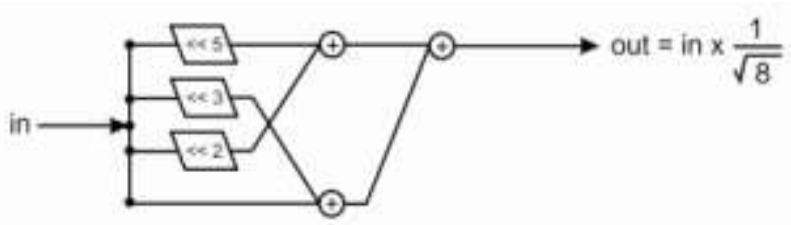

Figure 7 The $\mathrm{mCBE}_{0}$ block architecture.

\section{b. The $\mathrm{mCBE}_{1}$ Block}

Table 4 shows an illustration of the $\mathrm{mCBE}_{1}$ block design.

Table 4 Constant bit representation.

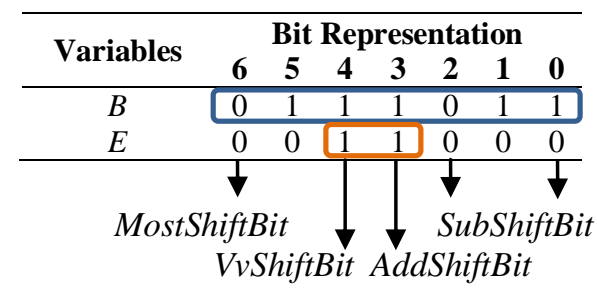

Then, we get the following architecture (Figure 8):

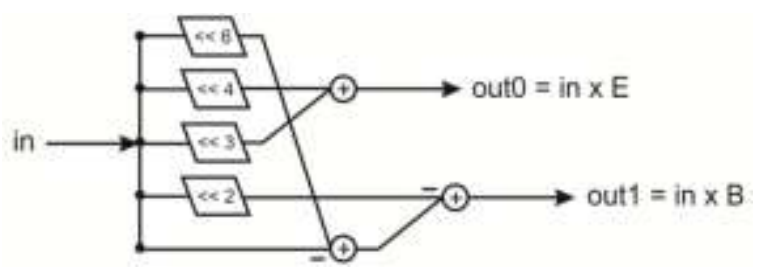

Figure 8 The $\mathrm{mCBE}_{1}$ block architecture.

\section{c. The $\mathrm{mCBE}_{2}$ Block}

Table 5 shows an illustration of the $\mathrm{mCBE}_{2}$ block design.

Table 5 Constant bit representation.

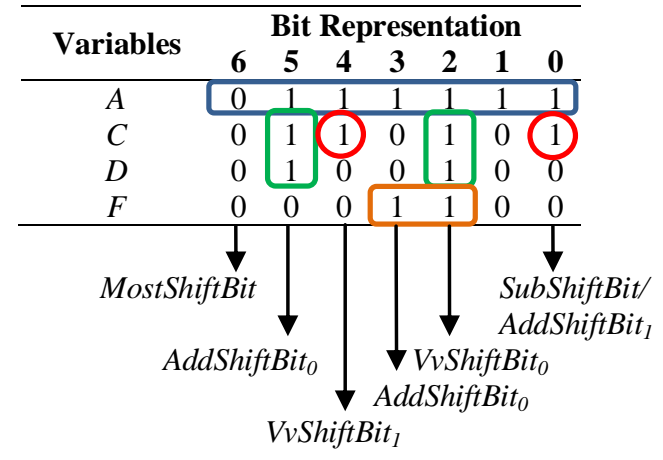


Then, we get the following architecture (Figure 9):

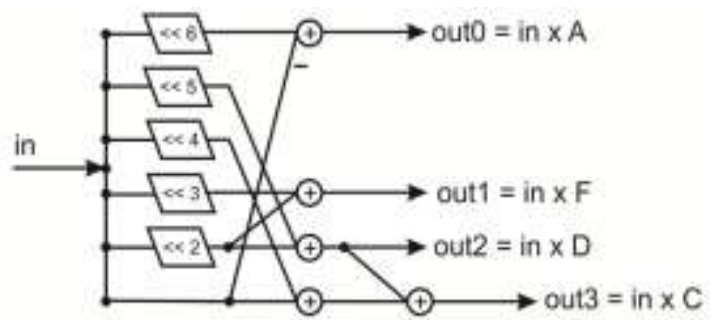

Figure 9 The $\mathrm{mCBE}_{2}$ block architecture.

In order to optimize the area consumption and retain a good image quality, we use some additional techniques. First, we expand the data word-length while the shifting process occurs in the $\mathrm{mCBE}_{0-2}$ blocks. Then, we cut the expanded data word-length at the last 7 bits to represent 20-bit fixed-point data, right after the 1D/2D-DCT/IDCT processes have been completed.

\subsection{Complete 8-Point 1D-DCT/IDCT Architecture}

The complete architecture is obtained by integrating the mCBE blocks with the remaining operations. The final 8-point 1D-DCT/IDCT architecture is shown in Figure 10-11.

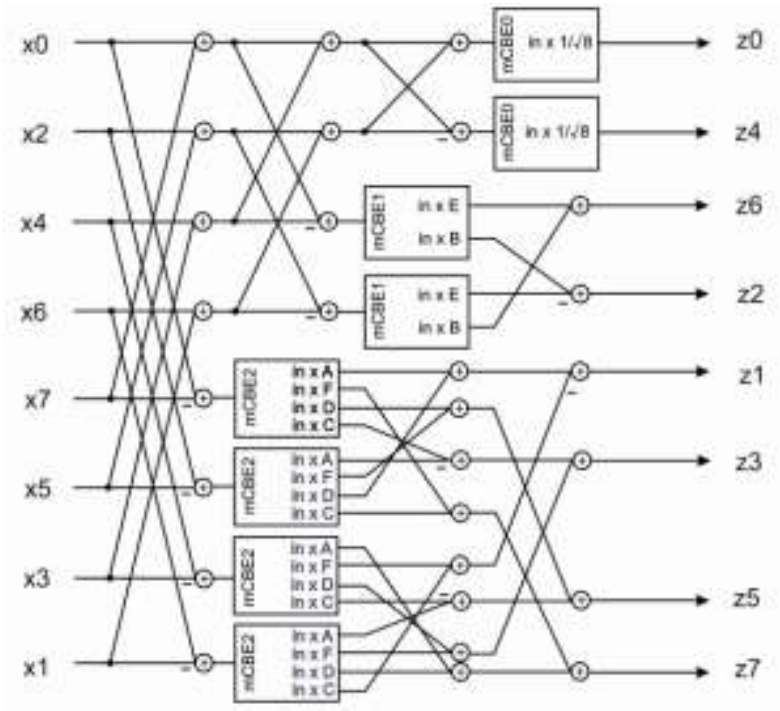

Figure 10 The proposed 8-point 1D-DCT architecture. 


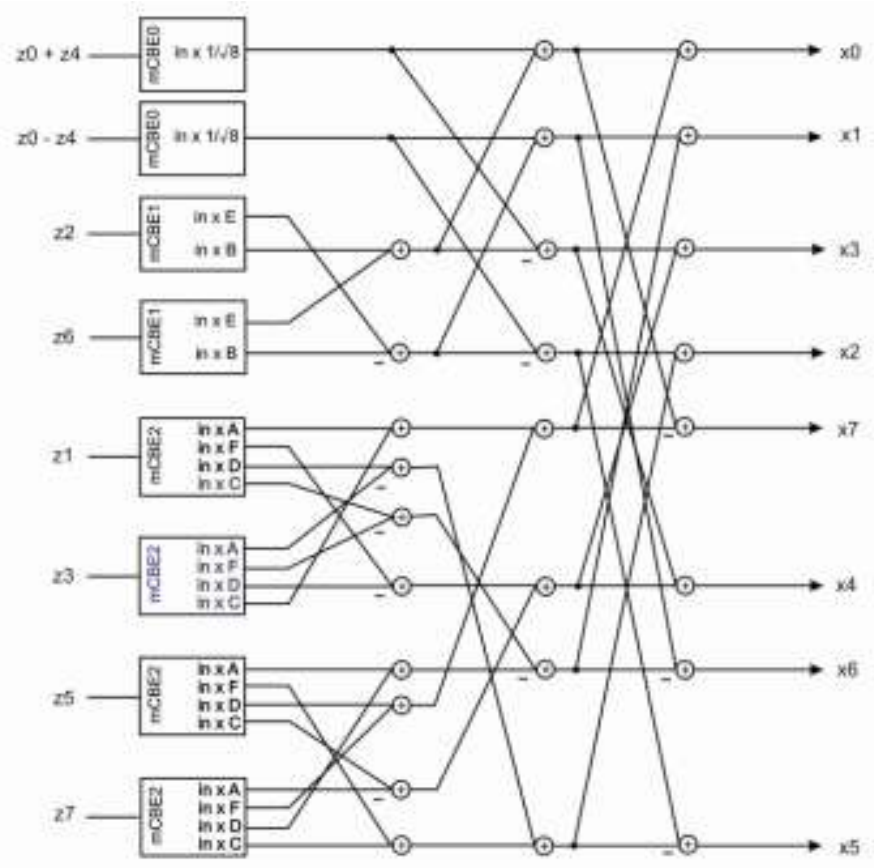

Figure 11 The proposed 8-point 1D-IDCT architecture.

From Figures 10 and 11, both designs (8-point 1D-DCT and 8-point 1D-IDCT) consist of 60 adders and 34 shifters. The 8 -point 1D-DCT has six stages and the 8-point 1D-IDCT has seven stages, where each stage is defined as the delay of one shifter or 2-input adder. The results fulfill the second and the third objective. The flow-graph architecture of each design has a less than 8- stage delay, without any iterations. Both designs only use shifter-adder components, so they have a low complexity and can be implemented easily in digital hardware. Furthermore, the designs can also easily be pipelined and modified for future improvement.

\subsection{Alternative Quantization-Dequantization Numbers}

In this paper we propose alternative quantization and dequantization numbers in order to simplify the hardware implementation without significantly reducing the image quality, based on the following considerations:

1. Image Quality Consideration : The proposed quantization-dequantization numbers can mostly retain and improve compressed-image quality compared to JPEG recommendation numbers. This can be seen from the retaining or increasing of the Peak-to-peak Signal to Noise Ratio (PSNR) value and the Mean Square Error (MSE) values. 
2. Low Complexity Hardware Implementation : The proposed quantizationdequantization numbers have to be implemented easily as shifter.

In order to retain image quality and get low complexity hardware, we adopt the recommended quantization numbers as reference and change the value of the numbers slightly compared to the recommended numbers, which are a power of 2.The proposed alternative quantization and dequantization numbers are:

$$
Q_{\text {LUMINANCE }}=Q_{\text {CHROMINANCE }}=\left[\begin{array}{cccccccc}
8 & 8 & 16 & 16 & 32 & 32 & 32 & 64 \\
8 & 16 & 16 & 32 & 32 & 32 & 64 & 64 \\
16 & 16 & 32 & 32 & 32 & 64 & 64 & 64 \\
16 & 32 & 32 & 32 & 64 & 64 & 64 & 128 \\
32 & 32 & 32 & 64 & 64 & 64 & 128 & 128 \\
32 & 32 & 64 & 64 & 64 & 128 & 128 & 128 \\
32 & 64 & 64 & 64 & 128 & 128 & 128 & 128 \\
64 & 64 & 64 & 128 & 128 & 128 & 128 & 128
\end{array}\right]
$$

For ease of reference, we call them FathQuantz numbers. If we use these numbers, there are consequences that we need to know. The design can be implemented easily as a shifter. However, the numbers are fixed, so we cannot make them change dynamically in the digital hardware, except if we design a supporting system for that case. In order to know the quality of the FathQuantz numbers, we examined them and compared the results with the reference numbers (JPEG recommendations, see Section 2.4). We use a comparison of PSNR and MSE values to show in how far there is a difference between the FathQuantz numbers and the reference numbers. The comparison results are shown in Figures 12-13.

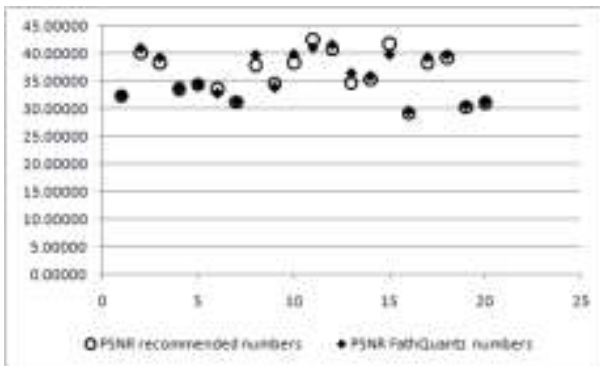

Figure 12 Comparison of PSNR values

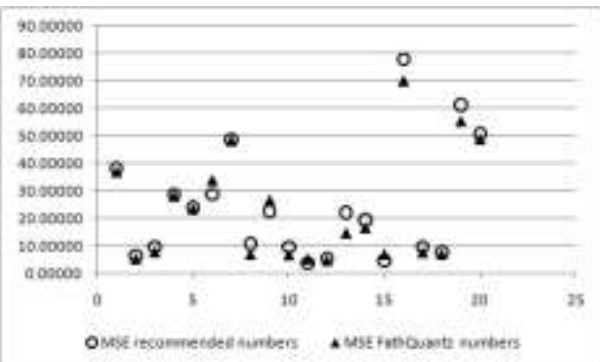

Figure 13 Comparison of MSE values.

In Figures 12 and 13, we can see that the PSNR and MSE values are not significantly different between the reference and the FathQuantz numbers. The. FathQuantz numbers can mostly retain the image quality compared to the reference numbers. Therefore, we can use the FathQuantz numbers as quantization-dequantization numbers. 


\subsection{Integration into the Complete Compression System}

Figure 14 shows the architecture of complete proposed compression system.

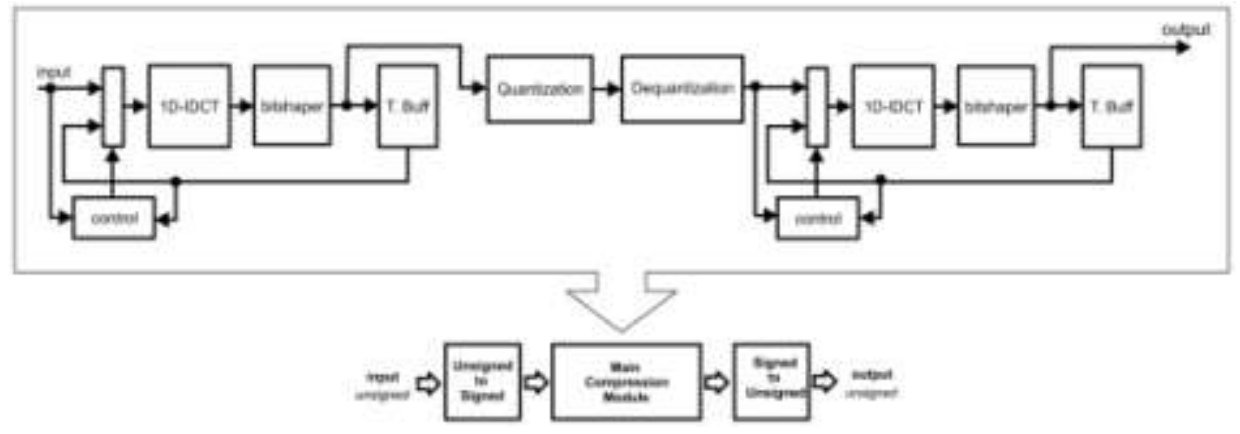

Figure 14 Complete proposed compression system.

\subsection{Pipelining Strategy}

As we all know, the best design conditions are built from low area consumption, high speed, and low latency. In our design, pipelining is purposely conducted to achieve a high throughput. Therefore, the pipelining strategy has to be conducted while taking area, speed, and latency into consideration. The pipelining strategy can be considered with two main conditions:

1. Low latency: In order to achieve a low latency, no pipeline is given.

2. High throughput : In order to achieve a high throughput, we can use a pipeline component to run a segmentation process. We can take several segments, such as: unsigned-to-signed data converter, 2D-DCT, quantization-dequantization, 2D-IDCT, and signed-to-unsigned data converter. If we want to add a number of pipelines to increase throughput, we can add them in the 1D-DCT/IDCT blocks to have segmentation in the process. However, we have to consider that adding pipelines also means adding area consumption and latency.

We have tested two scenarios to establish the design's boundaries. We tried the lowest latency design (without pipeline) and the highest throughput design (with the maximum amount of pipelines). We refer to the lowest latency design by its original name (e.g. DCT or IDCT) and to the highest latency design by adding "(p-)" in front of the name (e.g. p-DCT and p-IDCT). We will use the same references in the synthesis and benchmarks section.

\section{Design Synthesis and Benchmarks}

We have used Synopsys Design Vision with CMS-0.13 $\mu \mathrm{m}$ technology to synthesize the designs. 


\subsection{Parity Synthesis}

In order to make an easy comparison with other synthesis results, we also provide parity design. Parity design has a total 49 of 2 -input XOR elements and 21 stages (one stage is defined as the delay of one 2-input XOR element).

Table 6 Synthesis report of parity.

\begin{tabular}{cccccc}
\hline Design & $\begin{array}{c}\text { Total } \\
\text { Area }\end{array}$ & $\begin{array}{c}\text { Total } \\
\text { Data Arrival } \\
\text { Time }(\mathbf{n s})\end{array}$ & $\begin{array}{c}\text { Unit } \\
\text { Area }\end{array}$ & $\begin{array}{c}\text { Unit } \\
\text { Data Arrival } \\
\text { Time }(\mathbf{n s})\end{array}$ & $\begin{array}{c}\text { Total } \\
\text { Dynamic } \\
\text { Power }(\boldsymbol{\mu W})\end{array}$ \\
\hline Parity & 147 & 25.41 & 3 & 1.21 & 62.3955 \\
\hline
\end{tabular}

Hopefully, the parity design synthesis results in Table 6 will make it easier to compare our design with other designs.

\subsection{Synthesis of the mCBE-Based DCT/IDCT Architecture}

Table 7 presents the synthesis results of the mCBE-based designs.

Table 7 Synthesis report of mCBE-based DCT/IDCT architecture.

\begin{tabular}{rrrccc}
\hline \multicolumn{2}{c}{ Design } & Area & $\begin{array}{c}\text { Data Arrival Time } \\
(\mathbf{n s})\end{array}$ & $\begin{array}{c}\text { Total Dynamic Power } \\
(\mathbf{m W})\end{array}$ & $\begin{array}{c}\text { Latency } \\
\text { (cycles) }\end{array}$ \\
\hline DCT & 1D & 13787 & 90.00 & 16.02740 & 1 \\
IDCT & 1D & 14461 & 85.40 & 12.17250 & 1 \\
p-DCT & 1D & 43779 & 1.41 & 1.06960 & 6 \\
p-IDCT & 1D & 47024 & 1.41 & 0.67996 & 7 \\
p-DCT & 2D & 66179 & 1.41 & 1.04020 & 25 \\
p-IDCT & 2D & 69460 & 1.41 & 1.02410 & 27 \\
p-Compression & 143425 & 1.41 & 1.81550 & 55 \\
\hline
\end{tabular}

Notes:

DCT : DCT with mCBE Architecture

IDCT $\quad:$ IDCT with mCBE Architecture

p-DCT : Pipelined DCT with mCBE Architecture

p-IDCT : Pipelined IDCT with mCBE Architecture

p-Compression : Pipelined Complete Compression System with mCBE Arch.

From Table 7, we get that the designs with lowest latency have a slower data arrival time (about 60 times slower) than the designs with highest latency.

\subsection{Benchmarks}

In this section, we compare our design approach with other methods. Tables 8-9 show the benchmarks considering the multiplier design method and Tables 1011 show the benchmarks considering the 1D/2D-DCT/IDCT design approach. 
Table 8 8-point DCT benchmarks.

\begin{tabular}{cccc}
\hline Design & \multicolumn{3}{c}{ 8-points 1D-DCT } \\
\cline { 2 - 4 } Name & Area & Data Arrival Time (ns) & Total Dynamic Power (mW) \\
\hline Multiplier & 33865 & 203.43 & 31.7158 \\
Shift-Add & 18379 & 92.72 & 20.0942 \\
mCBE & 13787 & 90.00 & 16.0274 \\
\hline
\end{tabular}

Table 9 8-point IDCT benchmarks.

\begin{tabular}{cccc}
\hline Design & \multicolumn{3}{c}{ 8-points 1D-IDCT } \\
\cline { 2 - 4 } Name & Area & Data Arrival Time (ns) & Total Dynamic Power (mW) \\
\hline Multiplier & 33854 & 150.03 & 17.96460 \\
Shift-Add & 19277 & 85.72 & 13.79980 \\
mCBE & 14461 & 85.40 & 12.17250 \\
\hline
\end{tabular}

Table 10 1D-DCT/IDCT design approach benchmarks.

\begin{tabular}{ccccccc}
\hline \multirow{2}{*}{$\begin{array}{c}\text { Design } \\
\text { Name }\end{array}$} & Area & $\begin{array}{c}\text { 8-points 1D-DCT } \\
\text { Dime (ns) }\end{array}$ & $\begin{array}{c}\text { Latency } \\
\text { (cycles) }\end{array}$ & Area & $\begin{array}{c}\text { Data Arrival } \\
\text { Time (ns) }\end{array}$ & $\begin{array}{c}\text { Latency } \\
\text { (cycles) }\end{array}$ \\
\cline { 2 - 7 } Multiplier & 33865 & 203.43 & 1 & 33854 & 150.03 & 1 \\
Shift-Add & 18379 & 92.72 & 1 & 19277 & 85.72 & 1 \\
mCBE & 13787 & 90.00 & 1 & 14461 & 85.40 & 1 \\
Paper [4] & - & 3.33 & 45 & - & 3.33 & 67 \\
Paper [6] & - & - & 10 & - & - & - \\
p-mCBE & 43779 & 1.41 & 6 & 47024 & 1.41 & 7 \\
\hline
\end{tabular}

Table 11 2D-DCT/IDCT design approach benchmarks.

\begin{tabular}{|c|c|c|c|c|c|c|c|}
\hline \multirow[b]{2}{*}{$\begin{array}{l}\text { Design } \\
\text { Name }\end{array}$} & \multicolumn{3}{|c|}{ 8x8-points 2D-DCT } & \multicolumn{3}{|c|}{ 8x8-points 2D-IDCT } & \multirow[b]{2}{*}{$\begin{array}{c}\text { Synthesis } \\
\text { CMOS } \\
\text { Tech. } \\
(\mu \mathrm{m})\end{array}$} \\
\hline & Area & $\begin{array}{c}\text { Data } \\
\text { Arrival } \\
\text { Time } \\
\text { (ns) }\end{array}$ & $\begin{array}{l}\text { Latency } \\
\text { (cycles) }\end{array}$ & Area & $\begin{array}{c}\text { Data } \\
\text { Arrival } \\
\text { Time } \\
\text { (ns) }\end{array}$ & $\begin{array}{l}\text { Latency } \\
\text { (cycles) }\end{array}$ & \\
\hline Paper [3] & 214263 & 17.14 & - & - & - & - & Anam-0.18 \\
\hline Paper [4] & - & 3.33 & 172 & - & 3.33 & 178 & 0.35 \\
\hline Paper [7] & - & 6.02 & 45 & - & 6.02 & 45 & - \\
\hline p-mCBE & 66179 & 1.41 & 25 & 69460 & 1.41 & 27 & CMS-0.13 \\
\hline
\end{tabular}

Notes:

mCBE : DCT/IDCT design with mCBE Algorithm p-mCBE : Pipelined DCT/IDCT design with mCBE Algorithm Multiplier : DCT/IDCT design with multiplier

Shift-Add : DCT/IDCT design with shift-add operation without mCBE Alg.

Paper [n] : Proposed design in paper [n], $n=$ number of reference

(-) : No specific information 
From Tables 8 and 9, the proposed design has a smaller area and a faster data arrival time than the general multiplier method or the pure shift-add method. Moreover, the DCT/IDCT designs with mCBE algorithm are almost three times smaller and about two times faster than the multiplier-based architecture. The proposed design is also smaller and faster than a pure shift-add architecture. In Tables 10 and 11, we show the results of the comparison between the proposed design and other proposed designs, such as the designs from references [6], [7], [8] and [9]. The results show that our design can reach all three design objectives (small area, multiplierless, and low complexity). Moreover, the proposed design can achieve a high speed and relatively low latency compared to the other designs.

\section{$5 \quad$ Verification and Implementation}

In order to verify the functional performance of our design, we used MATLAB and Modelsim software (see Figures 15-20). First, we generated the image data file by using MATLAB. This file was read by our Verilog HDL codes and computed in Modelsim. The output of the compression system in Modelsim was written to a new file. Then, this file was read and reconstructed as a picture by MATLAB. In this step, we also compared the original image with the compressed one by using the PSNR and MSE computation method.

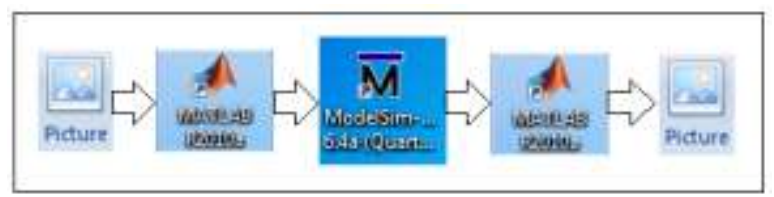

Figure 15 Functional verification flow.



Figure 16 Functional simulation process. 


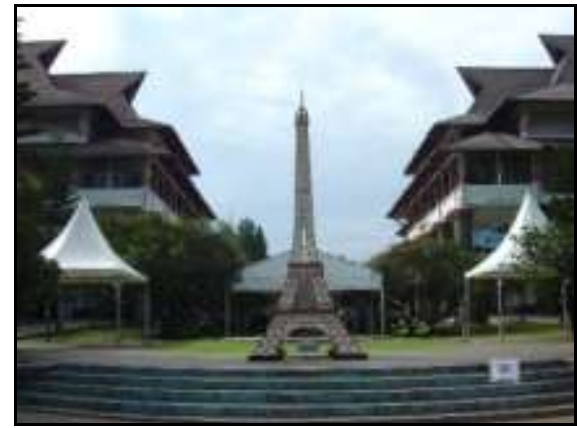

Figure 17 Original picture (77KB).

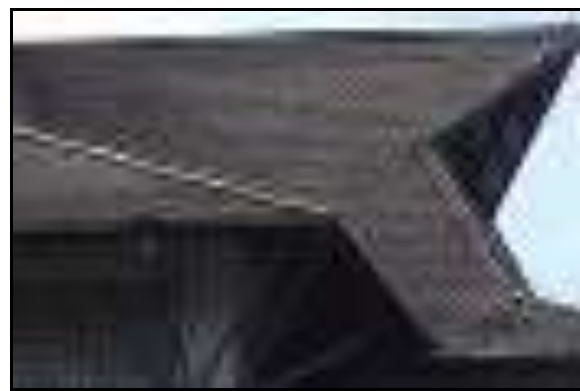

Figure 19 Cropped original picture.

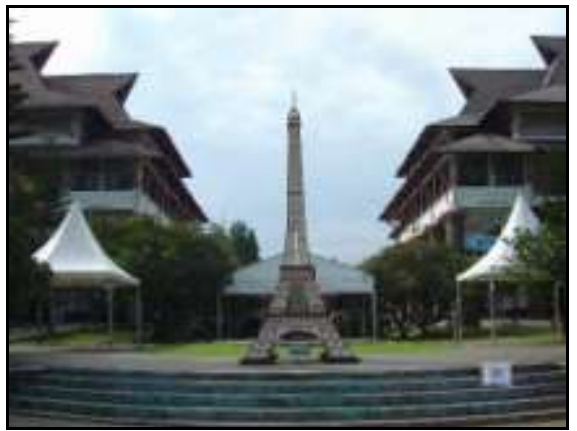

Figure 18 Compressed picture (37.2KB).



Figure 20 Cropped compressed picture.

The reconstructed compressed image data were computed using MATLAB to examine the PSNR and MSE values. Figure 18, the reconstructed image, has PSNR $=41.808 \mathrm{~dB}$ and MSE $=4.289$. From the example, we notice that the compressed image was slightly more blurred. Considering the PSNR and MSE values which we have obtained from several functional verifications, we conclude that our system can obtain a good compressed-image quality. This means that we have reached the first goal: the proposed design can operate well, producing a compressed image of good quality.

Our design has also been implemented in FPGA board Altera DE2 Cyclone II EP2C35F672C6. For FPGA implementation, we used the internal ROM to store the image data and compute them to give output. The computation output was presented using FPGA's LED to indicate whether the output data were valid or not. This FPGA implementation results show that the proposed design works well and gives valid output data. 


\section{Conclusion}

We conclude that our design can reach all three objectives successfully. The mCBE Algorithm can minimize the amount of shifter-adders to substitute multipliers. Besides that, our alternative quantization-dequantization numbers can mostly retain good compressed-image quality compared to JPEG recommendations. The results of this research show that the proposed 8-point 1D-DCT design has only six stages and the 8-point 1D-IDCT design has only seven stages. Here, we define one stage as equal to the delay of one shifter or 2input adder. By using the pipelining method, we can achieve a high-speed architecture with latency as trade-off consideration. This design has been synthesized and it can reach speeds of up to $1.41 \mathrm{~ns}$ critical path delay (709.22MHz).

\section{References}

[1] Salomon, D., Data Compression: The Complete Reference, 3rd Edition, New York: Springer-Verlag, 2004.

[2] Loeffler, C., Lightenberg, A. \& Moschytz, G.S., Practical Fast 1-D DCT Algorithms with 11-Multiplications, Proceedings ICASSP, 1989.

[3] Jeong, H., Kim, J. \& Cho, W.-k., Low-Power Multiplierless DCT Architecture Using Image Data Correlation, IEEE Transactions on Consumer Electronicsm, 2004.

[4] Ruiz, G.A., Michell, J.A. \& Buron, A., High Throughput ParallelPipeline 2-D DCT/IDCT Processor Chip, Journal of VLSI Signal Processing, 45, pp. 161-175, 2006.

[5] Heyne, B. \& Gotze, J., A Low Power and High Quality Implementation of The Discrete Cosine Transformation, Advance in Radio Science, 2007.

[6] Kim, B.-I. \& Ziavras, S.G., Low-Power Multiplierless DCT for Image/Video Coders, IEEE 13th International Symposium on Consumer Electronics, 2009.

[7] Subramanian, P. \& Reddy, A.S.C., VLSI Implementation of Fully Pipelined Multiplierless 2D DCT/IDCT Architecture for JPEG, Proceedings ICSP, 2010.

[8] LSI Design Contest Official Website, Okinawa, Japan, http://lsicontest.com/2011/shiyou_3e.html (1 December 2010).

[9] Sung, T.Y., Shieh, Y.S. \& Sun, M.J., A High-Throughput and MemoryEfficiency 2D DCT Architecture based on Cordic Rotation, The 23rd Workshop on Combinatorial Mathematics and Computation Theory. 